\title{
Nonlocal Description of the Nucleus-Nucleus Interaction
}

\author{
L. C. Chamon, D. Pereira, and M. S. Hussein \\ Instituto de Física, Universidade de São Paulo, C.P. 66318, 05315-970 São Paulo, SP, Brazil
}

M. A. Cândido Ribeiro

Department of Physics, University of Wisconsin, 1150 University Avenue, Madison, Wisconsin 53706

D. Galetti

Instituto de Física Teórica, Universidade Estadual Paulista, Rua Pamplona 145, 01405-900 São Paulo, SP, Brazil

(Received 2 July 1997)

A parameter-free nonlocal double-folding-inspired interaction is proposed for the nucleus-nucleus systems. Excellent reproductions of elastic scattering differential cross section data were obtained for several systems over a wide range of bombarding energies. Our results should be of value in the description of the scattering of other many-body systems. [S0031-9007(97)05269-1]

PACS numbers: 21.30.Fe, 21.60.-n, 24.10. $-\mathrm{i}, 25.70 . \mathrm{Bc}$

The mean field interaction between complex quantum many-body systems (nucleus-nucleus, cluster-cluster, etc.) is still an open question in current physics research. The study of this matter is a fundamental step in the understanding of many-body dynamics. In the nucleus-nucleus case, significant progress has been achieved concerning this question during the last decade [1], as a consequence of the measurement of accurate and extensive elastic scattering data at intermediate energies. Nuclear rainbow scattering, first observed in $\alpha$ systems [2-4] and later in light heavy ions [5-7], probes the nucleus-nucleus potential not only at the surface region but also at smaller distances, and ambiguities in the real part of the potentials have been removed. The resulting phenomenological interactions have significant dependence upon the bombarding energies. Some theoretical models have been developed to account for this energy dependence through realistic mean field potentials. Nowadays, the most successful models seem to be those based on the DDM3Y interaction [810] which is an improvement of the originally energyindependent double-folding potential [11]. But, in order to fit the data, the density- and energy-dependent DDM3Y potential needs a renormalization factor which besides being system dependent $[1,12]$ is still slightly energy dependent [1].

In this Letter we show, by an extensive description of elastic scattering data using an optical integro-differential equation, that the dependence on the bombarding energy of the real bare potential is mostly due to the intrinsically nonlocal nature of the effective one-body interaction. The real bare potential (by bare we mean the average, mean field, interaction with no coupled channels effects) is constructed using the folding model. It contains no adjustable parameters and is energy independent. The absorptive part is taken to be a three parameters WoodsSaxon interaction. We also supply a simple approach to obtain the local-equivalent energy-dependent potential.
Before we set the stage for the analysis of elastic scattering data, we first describe our theoretical model.

When dealing with nonlocal interactions, one is required to solve the integro-differential equation

$$
-\frac{\hbar^{2}}{2 \mu} \nabla^{2} \Psi(\vec{R})+\int U\left(\vec{R}, \vec{R}^{\prime}\right) \Psi\left(\vec{R}^{\prime}\right) d \vec{R}^{\prime}=E \Psi(\vec{R}),
$$

where, on physical grounds [13], the kernel function is taken to be symmetric: $U\left(\vec{R}, \vec{R}^{\prime}\right)=U\left(\vec{R}^{\prime}, \vec{R}\right)$. We take for $U\left(\vec{R}, \vec{R}^{\prime}\right)$ the following form motivated by the physics problem at hand

$$
\begin{aligned}
U\left(\vec{R}, \vec{R}^{\prime}\right)= & V\left(\vec{R}, \vec{R}^{\prime}\right)+i \delta\left(\vec{R}-\vec{R}^{\prime}\right) W(R, E) \\
& +\delta\left(\vec{R}-\vec{R}^{\prime}\right) V_{C}(R) .
\end{aligned}
$$

In our analysis, the Coulomb interaction, $V_{C}(R)$, was obtained using an expression for the double sharp cutoff folded potential [14] and the local energy-dependent imaginary potential, $W(R, E)$, was taken to have a WoodsSaxon form with three adjustable parameters.

Guided by the microscopic treatment of neutronnucleus scattering [15], we have assumed for the real nuclear interaction the Perey-Buck or Frahn-Lemmer ansatz [16,17]

$$
\begin{aligned}
V\left(\vec{R}, \vec{R}^{\prime}\right)= & V_{N L}\left(\frac{R+R^{\prime}}{2}\right) \frac{1}{\pi^{3 / 2} b^{3}} \\
& \times \exp \left[-\left(\frac{\left|\vec{R}-\vec{R}^{\prime}\right|}{b}\right)^{2}\right],
\end{aligned}
$$

where $b$ is the range of the Pauli nonlocality of the ion-ion interaction. Therefore, the nonlocality introduced in this way is a correction to the local model and in the $b \rightarrow 0$ limit Eq. (1) reduces to the usual Schrödinger differential equation. We should mention, at this point, that the use of the simple Gaussian shape to represent nonlocality in the nucleus-nucleus interaction has been justified, within the single-folding model, by Jackson and Johnson [18]. 
In a recent publication [19], we have shown that the values of $b$ can be obtained for light heavy-ion systems from the energy dependence of phenomenological potentials extracted from elastic scattering data analyses. These $b$ values were found to be in accord with the Jackson and Johnson [18] theoretical prediction: $b=$ $b_{0} m_{0} / \mu$, where $b_{0}$ is the nucleon-nucleus nonlocality parameter, $m_{0}$ is the nucleon mass, and $\mu$ is the reduced mass of the system. The value $b_{0}=0.85 \mathrm{fm}$ was well established by an extensive nucleon-nucleus elastic data analysis [16]. We have used the above system-dependent expression for $b$ in the interaction, Eq. (3).

We propose that $V_{N L}\left(\frac{R+R^{\prime}}{2}\right)$ is of the following foldinginspired form:

$$
\begin{gathered}
V_{N L}(\xi)=V_{\text {fold }}(R=\xi), \\
V_{\text {fold }}(R)=\int \rho_{1}\left(r_{1}\right) v\left(\vec{R}-\vec{r}_{1}+\vec{r}_{2}\right) \rho_{2}\left(r_{2}\right) d \vec{r}_{1} d \vec{r}_{2},
\end{gathered}
$$

where $\rho_{1}\left(r_{1}\right)$ and $\rho_{2}\left(r_{2}\right)$ are the nuclear densities of the colliding partners and $v(r)$ is the effective nucleonnucleon interaction. We point out that $V_{\text {fold }}(R)$ is assumed to have the local standard form of the double-folding potential [11]. We have used the well-known nucleonnucleon M3Y interaction

$$
\begin{aligned}
v(r)= & {\left[7999 \frac{e^{-4 r}}{4 r}-2134 \frac{e^{-2.5 r}}{2.5 r}\right] } \\
& -262 \delta(\vec{r}) \mathrm{MeV} .
\end{aligned}
$$

The pseudopotential, $-262 \delta(\vec{r}) \mathrm{MeV}$, describes the knock-on exchange collision at $10 \mathrm{MeV} /$ nucleon [12]. The two other Yukawa terms are responsible for the direct component of the interaction. We stress that $V\left(\vec{R}, \vec{R}^{\prime}\right)$ is a nonlocal exchange potential, since we have considered the exchange as the main source of the nonlocality. In our approach, we have not considered the intrinsic energy dependence in the effective nucleon-nucleon interaction since we associate all the observed energy dependence of the local equivalent potential to the parameter $b$. As has been discussed earlier within a microscopic treatment [15], such a hypothesis should be valid at energies up to $200 \mathrm{MeV} /$ nucleon. Therefore, the present data analysis is a further test to check our recent findings [19], which associate most of the observed energy dependence of the phenomenological local real potential to finite range exchange effects.

We turn next to the data analyses. After projecting over partial waves, we have solved numerically Eq. (1) by an iterative method. In order to test how general is our model, we have analyzed elastic scattering angular distributions for the ${ }^{12} \mathrm{C}+{ }^{12} \mathrm{C},{ }^{208} \mathrm{~Pb}$ and $\alpha+{ }^{12} \mathrm{C},{ }^{58} \mathrm{Ni}$ systems. These systems correspond to quite different nonlocality range parameters: $b=0.075 \mathrm{fm}\left({ }^{12} \mathrm{C}+{ }^{208} \mathrm{~Pb}\right)$, $b=0.14 \mathrm{fm} \quad\left({ }^{12} \mathrm{C}+{ }^{12} \mathrm{C}\right), \quad b=0.23 \mathrm{fm} \quad\left(\alpha+{ }^{58} \mathrm{Ni}\right)$, and $b=0.28 \mathrm{fm}\left(\alpha+{ }^{12} \mathrm{C}\right)$. For the first two sys- tems, the data are available at a wide energy range ( $1 \leq E_{\text {lab }} / A_{\text {proj }} \leq 200 \mathrm{MeV} /$ nucleon), whereas the last two systems represent typical cases of refractive scattering that have been studied in details in the early 1970s [2-4]. In the present analyses, we have used realistic microscopic nuclear densities as reported in the literature $[11,12,20]$. The only parameters that were allowed to vary were those of the absorptive Woods-Saxon imaginary potential. In Figs. 1 and 2 we show the angular distributions for the systems above at the following bombarding energies: $E_{\text {lab }}=$ $16,112,300,1016,1449$, and $2400 \mathrm{MeV} \quad\left({ }^{12} \mathrm{C}+{ }^{12} \mathrm{C}\right)$, $E_{\text {lab }}=420,1449$, and $2400 \mathrm{MeV}\left({ }^{12} \mathrm{C}+{ }^{208} \mathrm{~Pb}\right)$, and $E_{\text {lab }}=139 \mathrm{MeV}\left(\alpha+{ }^{12} \mathrm{C},{ }^{58} \mathrm{Ni}\right)$. The agreement between the theoretical calculations (solid lines) and the experimental data (from Refs. [4,5,21-26]) is impressive. The quality of the fits are at least as good as that of any other analyses using realistic potentials.

The resulting reaction cross section values are very similar to the already reported ones (Refs. $[1,4,5,21-26])$. The geometry of the imaginary potential $W(R)$ and the corresponding volume integral $J_{W}(E)$ behave as a function of $E$ quite smoothly. More specifically for the ${ }^{12} \mathrm{C}+{ }^{12} \mathrm{C}$ system, the behavior of $J_{W}(E)$ as a function of the bombarding energy is just like the one shown in Fig. (6.8) of Ref. [1]. To our knowledge, the results reported here correspond to a first attempt to reproduce light- and heavy-ion elastic angular distributions for such a variety of systems and such a wide energy range with completely parameter-free real interaction. Furthermore, the usual renormalization factor of the DDM3Y interaction is absent in our analyses. Thus, one reaches the conclusion that the widely used

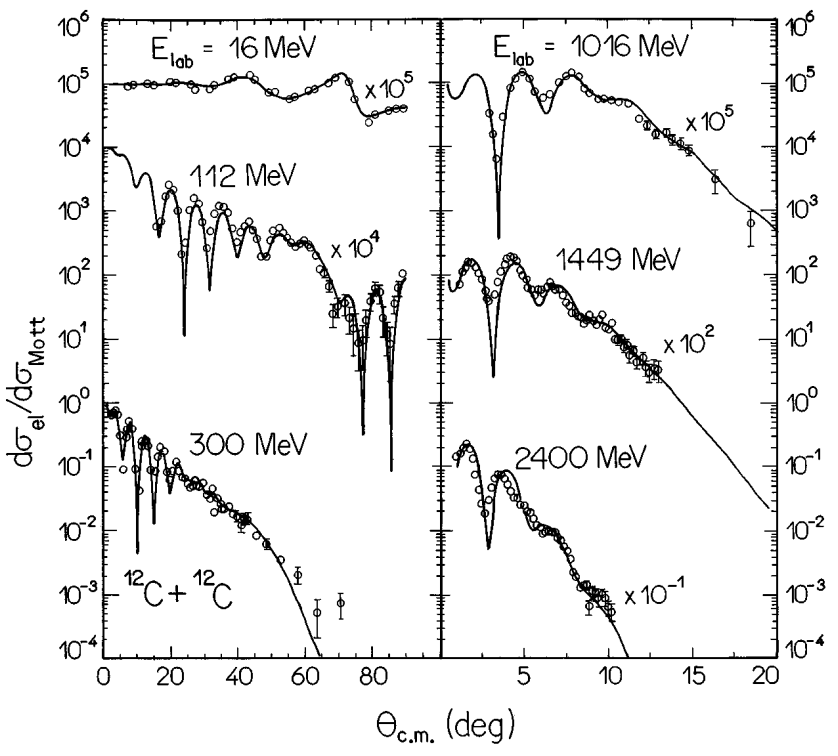

FIG. 1. Elastic scattering angular distributions for the ${ }^{12} \mathrm{C}+$ ${ }^{12} \mathrm{C}$ system at several bombarding energies as indicated. The data are from Refs. [5,21-24]. The solid lines correspond to nonlocal optical model calculations using an energyindependent and parameter-free real potential (see text). Note the change in the scattering angle scale. 


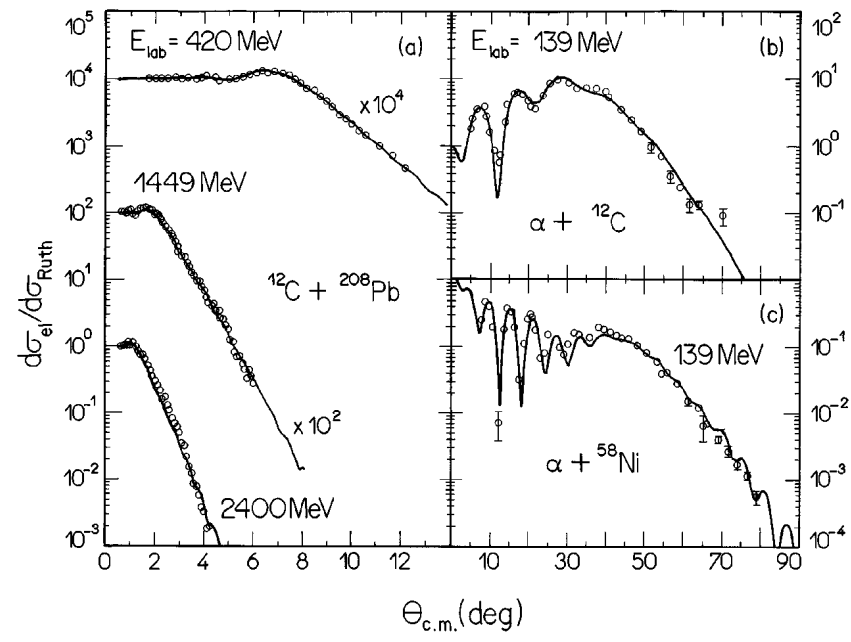

FIG. 2. The same as Fig. 1 for the systems ${ }^{12} \mathrm{C}+{ }^{208} \mathrm{~Pb}$ and $\alpha+{ }^{12} \mathrm{C},{ }^{58} \mathrm{Ni}$. The data are from Refs. [4,24-26]. Note the changes in the scales of both axes.

energy-dependent potential hides to some extent the underlying nonlocal interaction. We should mention, however, that there is room to have some density dependence in our model if a more optimized fit is undertaken. From the result we obtain, it is clear that this dependence is bound to be weak.

Because of the high values of orbital angular momentum involved in the calculations, the numerical solution of the integro-differential equation (1) is a very hard task for heavy-ion systems. Thus, it is desirable to develop a method to obtain the local equivalent potential (LEP) in order to calculate cross sections solving the usual Schrödinger equation. In the early 1960s, Perey and Buck [16] showed that the LEP is only weakly $\ell$ dependent and supplied an approximate relation to obtain that potential for neutron-nucleus systems. We have generalized [19] that expression to the nucleus-nucleus case, namely,

$$
\begin{aligned}
& \quad V_{\mathrm{LE}}(R ; E) \exp \left[-\gamma V_{\mathrm{LE}}(R ; E)\right] \\
& \quad \approx V_{\mathrm{fold}}(R) \exp \left\{-\gamma\left[E-V_{C}(R)-i W(R, E)\right]\right\},
\end{aligned}
$$

with $\gamma=\mu b^{2} / 2 \hbar^{2}$. The above equation can be solved for $V_{\mathrm{LE}}(R ; E)$ by the iterative method. Since for heavyion systems the value of $\gamma$ is very small (for ${ }^{12} \mathrm{C}+{ }^{12} \mathrm{C}$, $\gamma=0.0014 \mathrm{MeV}^{-1}$ ) we can appropriately expand (7) to obtain

$$
\begin{aligned}
V_{\mathrm{LE}}(R ; E) & \approx V_{\text {fold }}(R)[1-\gamma E] \\
& =V_{\text {fold }}(R)\left(1-\beta \frac{E_{\text {lab }}}{A_{\text {proj }}}\right),
\end{aligned}
$$

where $\beta=m_{0} b_{0}^{2} / 2 \hbar^{2}$ is a system-independent constant equal to $0.0086 \mathrm{MeV}^{-1}$. This linear behavior of the potential with the energy has been particularly observed in $\alpha$ nucleus scattering [18] and the experimentally extracted slope is in agreement with the theoretical $\beta$ value.
A better approximation, valid over a greater energy range, is obtained by neglecting the imaginary part of the potential and expanding the left hand side of Eq. (7) to lowest order in $\gamma$. The resulting quadratic equation may be solved to obtain

$$
\begin{aligned}
& V_{\mathrm{LE}}(R ; E) \\
& \quad \approx \frac{1-\sqrt{1-4 \gamma V_{\text {fold }}(R) \exp \left\{-\gamma\left[E-V_{C}(R)\right]\right\}}}{2 \gamma} .
\end{aligned}
$$

We point out that in the limit $b \rightarrow 0$ we find for both Eqs. (8) and (9) $V_{\mathrm{LE}}(R, E)=V_{\text {fold }}(R)$, which contains the exchange effects in the zero-range approximation as should be the case. We have calculated the local equivalent potential using Eq. (9) for the system ${ }^{12} \mathrm{C}+$ ${ }^{12} \mathrm{C}$ at three bombarding energies. The results are shown as solid lines in Fig. 3. For comparison, the folding potential, $V_{\text {fold }}(R)$, is also shown (dashed line). We can observe that the LEP have slightly different shapes than that of $V_{\text {fold }}(R)$. At high energies, we observe that $V_{\mathrm{LE}}(R, E)$ becomes factorized as $V_{\mathrm{LE}}(R) f(E)$ with $f(E)$ being roughly exponential.

We have also calculated the real part of the exact local equivalent potential through Eq. (1). The results convinced us that, as expected [16], the $\ell$ dependence of the LEP is very weak. The potential values arising from the exact calculation agree with those from Eq. (9) within about 3\%. We have also calculated elastic scattering angular distributions by solving the local Schrödinger differential equation using the approximate LEP from Eq. (9). The results are quite similar to those generated from the solution of the full integro-differential equation (1). It is important to mention that the parameters of the imaginary Woods-Saxon potential, $W(R, E)$, used in the exact calculations are somewhat different from those

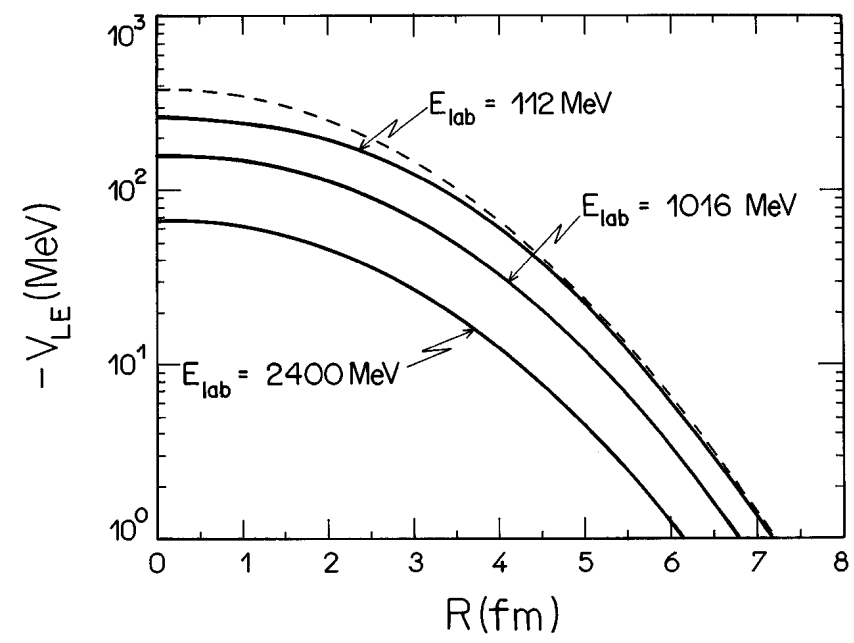

FIG. 3. The local equivalent potential (solid lines) for the ${ }^{12} \mathrm{C}+{ }^{12} \mathrm{C}$ system at three different bombarding energies. The dashed line corresponds to the double-folding energyindependent potential. 
used in the approximate ones, owing to the absence of $\operatorname{Im}\left[V_{\mathrm{LE}}(R, E)\right]$ in Eq. (9).

In conclusion, we have presented in this Letter a new model for the nucleus-nucleus interaction. The corresponding real potential is nonlocal and completely parameter-free. The local equivalent version of this interaction is angular-momentum independent and with a very simple energy dependence. Such potential reproduces the experimental elastic differential cross sections for a variety of systems and over a wide range of bombarding energies. Thus, the explicit consideration of the intrinsically quantum, finite range exchange nonlocality is of fundamental importance in the description of the nucleus-nucleus collision. Our results could be potentially important for the description of the scattering of other many-body systems.

A more detailed account of our work which will include an extensive comparison with the quasielastic scattering data of several other heavy ion systems will be published shortly.

M. A. C. R. is supported by FAPESP (Contract No. 96/ 3240-5) and all other authors are partly supported by CNPq. We thank G.R. Satchler for comments and suggestions.

[1] M.E. Brandan and G. R. Satchler, Phys. Rep. 285, 143 (1997).

[2] D. A. Goldberg and S. M. Smith, Phys. Rev. Lett. 29, 500 (1972).

[3] D. A. Goldberg and S. M. Smith, Phys. Rev. Lett. 33, 715 (1974).

[4] D. A. Goldberg, S. M. Smith, and G. F. Burdzik, Phys. Rev. C 10, 1362 (1974).

[5] H. G. Bohlen, M. R. Clover, G. Ingold, H. Lettau, and W. von Oertzen, Z. Phys. A 308, 121 (1982).

[6] H. G. Bohlen, X.S. Chen, J.G. Cramer, P. Frobrich, B. Gebauer, H. Lettau, A. Miezaika, W. von Oertzen, R. Ulrich, and Th. Wilpert, Z. Phys. A 322, 241 (1985).

[7] E. Stiliaris, H. G. Bohlen, P. Frobrich, B. Gebauer, D. Kolbert, W. von Oertzen, M. Wilpert, and Th. Wilpert,
Phys. Lett. B 223, 291 (1982).

[8] A. M. Kobos, B. A. Brown, P. E. Hodgson, G. R. Satchler, and A. Budzanowski, Nucl. Phys. A384, 65 (1982).

[9] A. M. Kobos, B. A. Brown, R. Lindsay, and G. R. Satchler, Nucl. Phys. A425, 205 (1984).

[10] D. T. Khoa, W. von Oertzen, and A. A. Ogloblin, Nucl. Phys. A602, 98 (1996).

[11] G. R. Satchler and W. G. Love, Phys. Rep. 55, 183 (1979).

[12] M. El-Azab Farid and G. R. Satchler, Nucl. Phys. A438, 525 (1985).

[13] R. Peierls and N. Vinh Mau, Nucl. Phys. A343, 1 (1980).

[14] R. M. Devries and M. R. Clover, Nucl. Phys. A243, 528 (1975).

[15] W. Bauhoff, H. V. von Geramb, and G. Palla, Phys. Rev. C 27, 2466 (1983).

[16] F. Perey and B. Buck, Nucl. Phys. 32, 253 (1962).

[17] W.E. Frahn and R.H. Lemmer, Nuovo Cimento 5, 1564 (1957).

[18] D. F. Jackson and R. C. Johnson, Phys. Lett. 49B, 249 (1974).

[19] M. A. Cândido Ribeiro, L. C. Chamon, D. Pereira, M. S. Hussein, and D. Galetti, Phys. Rev. Lett. 78, 3270 (1997).

[20] L. C. Chamon, D. Pereira, E. S. Rossi, Jr., C. P. Silva, H. Dias, L. Losano, and C. A. P. Ceneviva, Nucl. Phys. A597, 253 (1996).

[21] W. Treu, H. Frohlich, W. Galster, P. Duck, and H. Voit, Phys. Rev. C 22, 2462 (1980).

[22] R. G. Stokstad, R. M. Wieland, G. R. Satchler, C. B. Fulmer, D. C. Hensley, S. Raman, L. D. Rickersten, A. H. Snell, and P. H. Stelson, Phys. Rev. C 20, 655 (1979).

[23] M. Buenerd, A. Lounis, J. Chauvin, D. Lebrun, P. Martin, G. Duhamel, J. C. Gondrand, and P. de Saintignon, Nucl. Phys. A424, 313 (1984).

[24] J. Y. Hostachy, M. Buenerd, J. Chauvin, D. Lebrun, Ph. Martin, J. C. Lugol, L. Papineau, P. Roussel, N. Alamanos, J. Arviex, and C. Cerruti, Nucl. Phys. A490, 441 (1988).

[25] C. C. Sahm, T. Murakami, J. G. Cramer, A. J. Lazzarini, D. D. Leach, D. R. Tieger, R. A. Loveman, W. G. Lynch, M. B. Tsang, and J. Van der Plicht, Phys. Rev. C 34, 2165 (1986).

[26] S. M. Smith, G. Tebell, A. A. Cowley, D. A. Goldberg, H. G. Pugh, W. Reichart, and N.S. Wall, Nucl. Phys. A207, 273 (1973). 